\title{
Improvements on full-profile fitting pendent drop method used for surface tension measurement
}

\author{
Siwei $\mathrm{Li}^{\mathrm{a}}$, Jinglong $\mathrm{Li}^{\mathrm{b}}$ \\ Department of Precision Instrument, Tsinghua University, Beijing 100084, China. \\ alswdlswxs@126.com, 'rijinlon@gmail.com
}

Keywords: surface tension, full-profile fitting method, pendent drop.

\begin{abstract}
Surface tension is an important parameter of liquid surface properties. In order to improve the accuracy and stability of the parameter measurement, full-profile fitting pendent drop method is utilized in this article, and algorithm to determine the initial value in two-dimensional optimization is improved. Moreover, the error function is modified via a weighting factor, which reduces the influence of droplet profile deviation point to the surface tension measurement. At $25^{\circ} \mathrm{C}$ and a standard atmospheric pressure, the improved full-profile fitting method is used to calculate the surface tension of water and ethanol. The final results show that the stability and repeatability of the improved full-profile fitting pendent drop method is much better than the previous method, which effectively verifies the feasibility and superiority of the improved method.
\end{abstract}

\section{Introduction}

Surface tension is of great significance in chemical, thermal, energy and other fields. It is directly related to the quality and performance of material. Therefore, studying the surface properties of material and improving it by measuring the surface tension, are particularly critical.

Surface tension measurements are mainly divided into two categories, dynamic method and static method [1]. Dynamic method mainly consists of capillary wave method and oscillation jet method. Static method include as drop weight, maximum bubble pressure, ring [2, 3], pendant drop method [4], sessile drop method [5], wave scattering method [6], electromagnetic levitation method [7], etc. Among them, the pendant drop method has become more and more prevalent because of its simplicity of measuring equipment, applications in high temperature conditions, and good accuracy of measurement.

Pendant drop method is based on Young-Laplace equation, and describes the balance of gravity and surface tension. In 1938, Andreas proposed to measure the size at two extreme positions of the pendant drop to calculate the liquid surface tension, naming selected plane method [8]. Although the operation is simple, the result is of low accuracy. With the development of computer and image processing, full-profile fitting method, using Multi-point or all of the droplet profile point to calculate the surface tension is developed and applied rapidly, which greatly improves the measuring accuracy of surface tension. The algorithm using full-profile fitting method to calculate surface tension mainly concludes one dimensional optimization [8], coordinate rotation method, simplex method [9], and Newton iteration method [10].

In this paper, according to the principle of full-profile fitting pendent drop method, we discussed and improved the algorithm for more accurate and stable measuring results. Not only the algorithm to determine the initial value is improved, but the error function is also modified via a weighting factor. By measuring the surface tension of water and ethanol respectively, the feasibility of this improved method is verified.

\section{Surface tension calculated from the drop profile}

The basic principle of pendant drop method is: when the droplet is still hanging in the capillary burette, the droplet profile depends on the balance of gravity and surface tension. Therefore, surface tension of the liquid can be calculated by measuring the droplet outer profile [11]. 
For any curved interface, the pressure difference across the interface and curvature of the interface can be expressed as:

$$
\Delta P=\gamma\left(\frac{1}{R_{1}}+\frac{1}{R_{2}}\right)
$$

Eq. (1) is the classical Laplace equation, $\gamma$ is interfacial tension of the liquid, $R_{1}$ and $R_{2}$ are the two principal radius of curvature of the interface. $\Delta P$ is the pressure difference across the interface. When the liquid is under gravity only with no other external conditions:

$$
\Delta P=\Delta P_{0}+\Delta \rho g z
$$

Where $\Delta P_{0}$ is the pressure difference at the apex of pendant drop, $\Delta \rho$ is the density difference between the fluid and gas, $g$ is the gravitational acceleration constant, $z$ is the droplet spatial position (Fig. 1). For the apex of droplet, it satisfies the expression below because of the symmetry: $R_{1}=R_{2}, \Delta P_{0}=2 \gamma / R_{0}$

As for the pendent drop, according to the geometry shown in Fig. 1, and Eqs. (1)-(2), we can nondimensionalizing $x, z, \theta$ by the radius of curvature at the apex, and then recast three differential equations as follows [12]:

$$
\left\{\begin{array}{l}
\frac{d \theta}{d S}=2-\beta Z-\frac{\sin \theta}{X} \\
\frac{d X}{d S}=\cos \theta \\
\frac{d Z}{d S}=\sin \theta
\end{array}\right.
$$

where $\beta=(\Delta \rho) \mathrm{gR}_{0}^{2} / \gamma, \quad X=x / R_{0}, \quad Z=z / R_{0}, \quad S=s / R_{0}$. Coordinate parameters $x, z, \theta$ and arc length $S$ refer to Fig. 1. Therefore, when the shape factor $\beta$ and the radius of curvature at the apex of the droplet $R_{0}$ are known, surface tension can be solved. Due to the limitations of computer application and image processing skills in the past, extracting the complete profile of droplet is difficult, so at that time, measuring the size at two extreme positions of droplet is widely applied to calculate the surface tension of the liquid, namely selected plane method. With the rapid development of computer and image processing, pendant drop method gradually shows its advantages over selected plane method $[13,14]$. By extracting multiple or all of the points on the outer profile of the droplet, and then fitting the curve, surface tension can be solved.

From Eq. (3), we know that, when given an initial value and a fixed step, according to Newton iteration and Runge-Kutta, each shape factor $\beta$ corresponds to a certain fitting curve. By changing the value of $\beta$ within a certain range, we can get a set of fitting curve results, as shown in Fig. 2.

Suppose the coordinate of droplet real profile point is $\left(x_{i}^{\prime}, z_{i}^{\prime}\right)$, and define the single point error function as:

$$
e_{i}=\frac{1}{2}\left[\left(\mathrm{x}_{i}-x_{i}^{\prime}\right)^{2}+\left(\mathrm{z}_{i}-z_{i}^{\prime}\right)^{2}\right]
$$

Then the real profile and the theory profile error function is:

$$
E=\sum_{i=1}^{N} e_{i}
$$

Where $\mathrm{x}_{i}=R_{0} X_{i}, \mathrm{z}_{i}=R_{0} Z_{i}$. When Eq. (5) meets the convergence condition as the objective function, we solve $\beta$ and $R_{0}$, then the liquid surface tension can be calculated. Since the pendant drop is symmetrical, we just choose right profile to calculate when solving the error function, which will double the computational efficiency. 


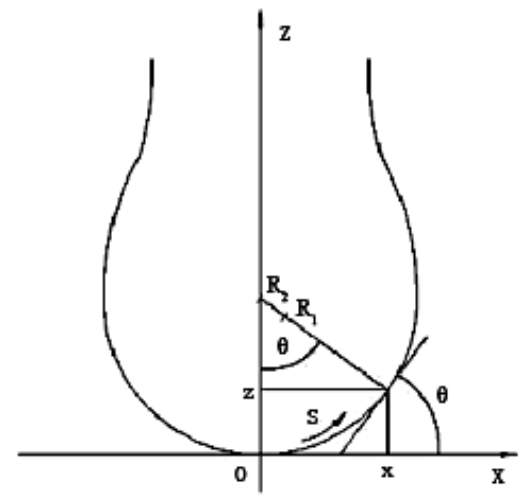

Fig. 1 Geometric schematic diagram of pendant drop

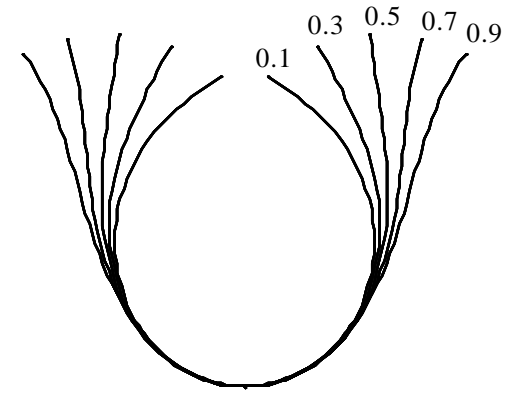

Fig .2 Fitted curves with different $\beta$

\section{Improvements of Surface tension calculation}

In the algorithm utilizing full-profile to measure the liquid surface tension, the one-dimensional optimization calculation is simple, but the measurement error is larger; Newton iterative method requires first-order partial derivative, second-order partial derivative and Hessian matrix, so its calculation process is relatively complex. Moreover, the initial values have a large effect on the calculation result; Coordinate rotation method and the simplex method need to judge whether calculation results meet the convergence conditions, when the convergence conditions meet, loop is end, and the variable is solved. Currently, initial determination of coordinate rotation method and simplex method is based on the selected plane method.

\subsection{Improvements on variable initial values calculation.}

To reduce the amount of calculation, we need to give an appropriate variable initial values and variable range when utilizing Coordinates rotation method to solve the surface tension, since the selected plane method just measure the size at two extreme positions of droplet, and then uses empirical equations to solve, it has a low accuracy. Therefore, another method is proposed to solve the variable initial value.

First, based on the actual droplet image, the least square method is used to fit the bottom profile of the droplet, which directly solves the radius of curvature at the apex of the droplet, as well as the initial value of variable $R_{0}$. Assume $R_{0}$ is a constant, then single variable optimization is done for $\beta$. Let $\beta$ change from 0.05 to 0.50 , with an interval value as 0.001 , we can get a set of theoretical fitting curve using fourth-order Runge-Kutta method. Combined with the real droplet profile point, Eq. (5) is used to solve the error function of theoretical profile and real profile. Initial value of variable $\beta$ can be obtained when the error function reaches its minimum value.

\subsection{Modify the error function}

In the process of droplet profile points extraction, there may be individual points deviate from the whole profile, which will lead to the objective function error, and make results of the final variables inaccurate, as shown in Fig. 3.

Assume curve $\mathrm{b}$ is the droplet real profile, curve a and $\mathrm{c}$ is the theoretical fitting profile when $\beta=0.1$ and $\beta=0.2$. When the droplet real profile is smooth and accurate, using the Eq. (5) to calculate the error function, we get the error of theoretical fitting profile a with real profile $b, E_{a}=0.0500$, and error of theoretical fitting profile c with real profile $b, E_{c}=0.0317, E_{c}<E_{a}$. Thus, we concluded that curve c fits the real profile better, $\beta=0.2$ is the final variable value.

When the droplet profile has an error, individual points deviate from the whole profile, thus when using the Eq. (5) to solve the error function, we get error $E_{a}=0.0423$, and error $E_{c}=0.0434, E_{a}<\mathrm{E}_{c}$. So we concluded that curve a fits the real profile better, $\beta=0.1$.

Compare the two cases above, we find that profile deviation points may cause an error of function (5) calculation, and finally affect the variable values. Therefore, we modified the error function via a weighting factor, which can reduce the influence of deviation point to the result. The weighting function is [15]: 


$$
w(t)=(1-t)^{2}+0.125
$$

Where $t=d_{i} / d_{\max }, d_{i}=\sqrt{\left(\mathrm{x}_{i}-x_{i}^{\prime}\right)^{2}+\left(\mathrm{z}_{i}-z_{i}^{\prime}\right)^{2}}, d_{\max }$ is the maximum of $d_{i}, \quad\left(\mathrm{x}_{i}, z_{i}\right)$ is the theoretical profile point coordinate, $\left(x_{i}^{\prime}, z_{i}^{\prime}\right)$ is the real profile point coordinate.

$$
\begin{aligned}
& e_{i}(2)=\frac{1}{2}\left\{w(\mathrm{t})\left[\left(\mathrm{x}_{i}-x_{i}^{\prime}\right)^{2}+\left(\mathrm{z}_{i}-z_{i}^{\prime}\right)^{2}\right]\right\} \\
& E(2)=\sum_{i=1}^{N} e_{i}(2)
\end{aligned}
$$

For the case that deviation points exist, we use the weighted error function (8) to calculate again, the result is $E(2)_{a}=0.0115, E(2)_{c}=0.0110, E(2)_{c}<E(2)_{a}$, it means that curve c fits the real profile better, so $\beta=0.2$.This result is consistent with the conclusion when real profile smooth and accurate, it proves that this algorithm can effectively reduce the influence of deviation point and make the final variable calculation more accurate.
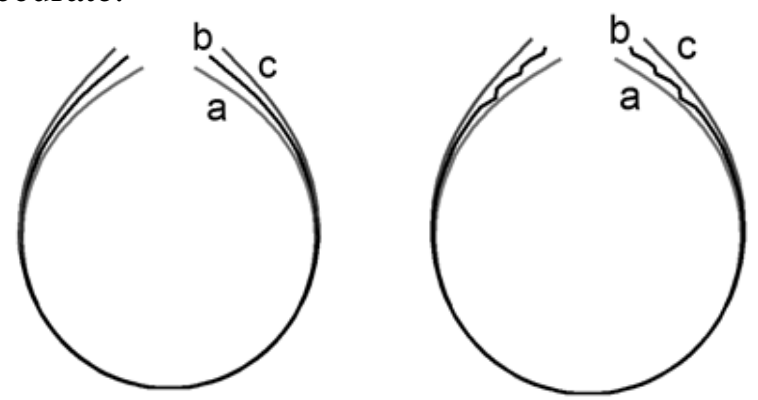

Fig .3 Fitting profile with deviation point

\subsection{The improved algorithm of surface tension}

First, utilize the variable initial values calculation in part 2. to get $R_{0}$ and $\beta$, according to the fitting error under initial condition, we determine the range of variable $R_{0}$ is $R_{0} \pm 0.5 \mathrm{~mm}$, range of $\beta$ for water and ethanol is $0.3-0.4$ and $0.45-0.55$ respectively.

Second, utilize the method of coordinate rotation to do single variable optimization for $R_{0}$ and $\beta$. We use Eq. (5) to solve the error function for each group variables, and judge whether it meets the error condition preset $\left(E<\xi\right.$ ), then store all the value of $R_{0}$ and $\beta$ which meet the error condition, at the end of the cycle, a set of corresponding sequences $R_{0}$ and $\beta$ can be obtained.

Third, to reduce the influence of deviation points, for the sequences $R_{0}$ and $\beta$ obtained above, we utilize the modified error function (8) to calculate again. Then select new sequences $\quad R_{0}$ and $\beta$ which meet the modified error condition, finally we use the equation $\gamma=(\Delta \rho) \mathrm{gR}_{0}^{2} / \beta$ to solve the surface tension $\gamma$.

\section{Results}

In this paper, we collect water and ethanol droplet image, and use original full-profile fitting method and improved method to calculate its surface tension respectively. The results of water surface tension measurements are shown in Fig. 4 and ethanol results in Fig. 5. 


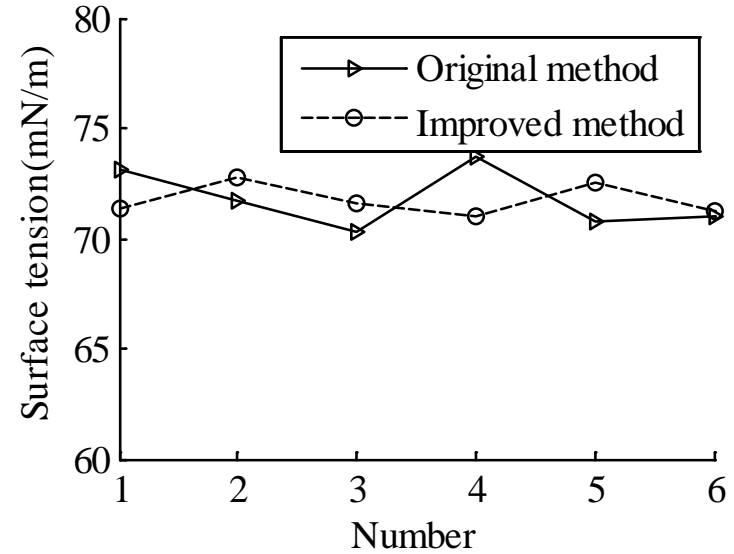

Fig. 4 Water surface tension measurement

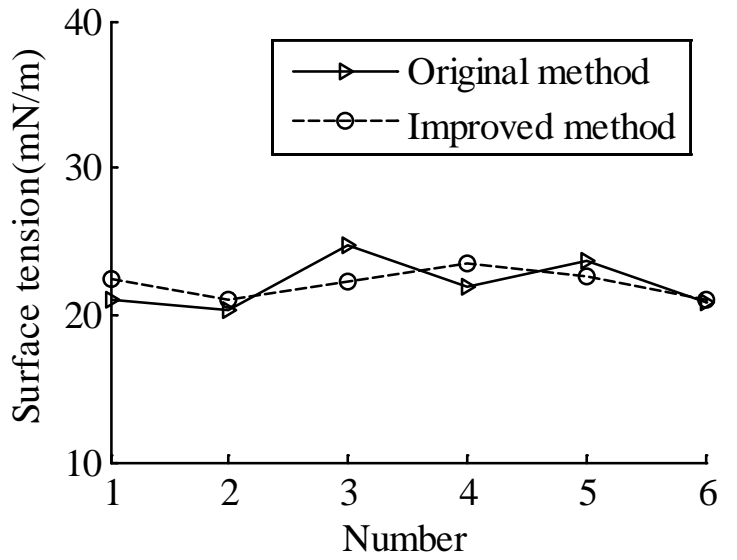

Fig .5 Ethanol surface tension measurement

Analyze the results of the six groups (Fig. 4), we find that the average surface tension of water calculated by original full-profile fitting method is $71.7638 \mathrm{mN} / \mathrm{m}$, the standard deviation of six experimental measurements is $1.3569 \mathrm{mN} / \mathrm{m}$.; The average surface tension calculated by improved method is $71.7552 \mathrm{mN} / \mathrm{m}$, the standard deviation is $0.7509 \mathrm{mN} / \mathrm{m}$. Compared to the original method, the improved method make the standard deviation decrease by $44.66 \%$, while the average difference is $0.01 \%$. It shows that the improved method remains the measurement accuracy, but stability is improved obviously.

Similarly, in Fig. 5, we analyze ethanol surface tension measurement data, the average surface tension calculated by original full-profile fitting method is $22.0915 \mathrm{mN} / \mathrm{m}$, the standard deviation is $1.7393 \mathrm{mN} / \mathrm{m}$; the average surface tension calculated by improved method is $22.1350 \mathrm{mN} / \mathrm{m}$, and the standard deviation is $0.9963 \mathrm{mN} / \mathrm{m}$. Compared with the original algorithm, the average difference is $0.20 \%$, while the standard deviation decrease by $42.72 \%$. It proved once again that when the measurement accuracy is almost consistent, stability is improved obviously by improved method.

\section{Conclusions}

Full-profile fitting pendent drop method, which is used to calculate the surface tension, is studied in this paper. Improvements based on the initial values and the error function are made. Experiments on water and ethanol are done to calculate the surface tension. The experimental results show that compared with the previous method, the measurement results of improved full-profile fitting pendent drop method are almost consistent, but the stability is obviously enhanced. It verifies that the improved method has better repeatability and provides theoretical support for the extensive use of this method.

\section{References}

[1]. Dongxia Yin, Peisheng Ma, Shuqian Xia. Progress on methods for measuring surface tension of liquids. Bulletin of Science and Technology. Vol. 23 (2007) No. 3, p. 424-425.

[2]. Chuanjun Li, Long Chen, Zhongming Ren. Application of ring method to measure surface tensions of liquids in high magnetic field. Review of Scientific Instruments. Vol. 83 ( 2012), p. 1-2.

[3]. Xuejun Wang, Xien Hu, Weimin Bao. Studies on automatical measurement of surface tension employing the ring method, Instrument Technique and Sensor. Vol. 11 (1997), p. 15-16.

[4]. Moradian A, Mostaghimi J. High Temperature Surface Tension Measurement. IEEE Transactions on Plasma Science. Vol. 33 (2005) No. 2, p. 410-411.

[5]. Jianfeng Fan, Zhangfu Yuan, Jiajun Ke. Development in measuring surface tension of high temperature molten liquid. Chemistry. Vol. 11 (2004), p. 804-805. 
[6]. Osada R, Hoshino T, Okada K, et al. Surface tension of room temperature ionic liquids measured by dynamic light scattering . The Journal of Chemical Physics. Vol. 130 (2009), p. 1-5.

[7]. H P Wang, J Chang, B Wei. Measurement and calculation of surface tension for undercooled liquid nickel and its alloy. Journal of Applied Physics. Vol. 106 (2009), p. 1-3.

[8]. Kevin Mohan: Measuring Interfacial Tension with the Pendant Drop Method (Bachelor's Degree, Georgia Institute of Technology, America, 2014). p. 11-16.

[9]. Andreas J M, Hauser E A, Tucker W B. Boundary tension by pendant drops. Journal of Biomechanics. Vol. 42 (1938) No. 8, p. 1001-1019.

[10]. Qiao Ning, Zhiqiang Zhu, Xutao Lv, et al. Determine the surface tension and contact angle of drop by image processing method. Chinese Journal of Space Science. Vol. 28 (2008) No. 1, p. 75-77.

[11]. Yaoyuan Chang, Mingya Wu, Yilin Hung. Accurate surface tension measurement of glass melts by the pendant drop method. Review of Scientific Instruments. Vol. 82 (2011), p. 3-4.

[12]. Xiaofeng Li, Zhirong Chen, Haihua Pan. Determination of dynamic interfacial tension by computer-aided pendant drop digitization. Journal of Chemical Industry and Engineering. Vol. 52 (2001) No. 6, p. 546.

[13]. Ali A, Badran. Oscillating pendant drop: A method for the measurement of dynamic surface and interface tension. Review of Scientific Instruments. Vol. 57 (1986), p. 259-261.

[14]. Fangyang Ai, Shengshan Bi, Jiangtao Wu. Surface tension experiment system with full-profile fitting pendant drop method. Physics Experimentation. Vol. 35 (2015) No. 5, p. 2-4.

[15]. Foley T A. Scattered data interpolation and approximation with error bounds. Computer Aided Geometric Design. Vol. 163 (1986) No. 3, p. 163-177. 